\title{
Preanodized Screen Printed Carbon Electrode for Detection of Linalool using Three Terminal Network
}

\author{
Abhishruti Bhuyan, Bipan Tudu, Rajib Bandyopadhyay, Sudarshan Gogoi, Amarprit Singh
}

\begin{abstract}
Linalool is a very important flavouring compound found in plants which is used in food and beverages. Linalool has been traditionally detected by analytical instruments such as gas chromatography (GC) coupled with mass spectroscopy(MS) which are not suitable for routine tests. For fast and low cost detection of chemical compounds electrochemial sensors are most suitable. Screen printed carbon electrode (SPCE) is one of the most popular and low cost device used for detection of chemical compounds. In this article we present the detection of linalool using a low cost preannodized commercial screen printed carbon electrode (SPCE). Traditionally electrochemical sensors are used in two terminal mode, however three terminal analysis of electrochemical sensors are found to be more rationale and accurate. In this paper we have analyzed detection of linalool by an advanced three terminal analysis. First we have performed cyclic voltammetry $(C V)$ of the SPCE which showed clear oxidation peaks at different concentration of linalool. The input-output data of the $\mathrm{CV}$ has been used for analysis of the impedance of the SPCE.The impedance model of the SPCE was estimated by autoregressive moving average with exogenous inputs(ARMAX) modelling technique using the $C V$ data. The three terminal impedance fitting revealed the values of electrical parameters and the parasitic elements at different linalool concentration. The stability limits of the SPCE was also determined from the pole-zero and Nyquist plots of the estimated models. Impedance behaviour to frequency of the SPCE was further analyzed by impedance $\operatorname{plot}\left(Z v s-Z^{\prime}\right)$ from which we are able to relate the $C V$ scan rate to the impedance of the SPCE. Finally the sensitivity and repeatability of the SPCE was determined using a measurement circuit.
\end{abstract}

Keywords: Linalool detection, SPCE, three terminal analysis, ARMAX modeling, parasitic elements.

\section{INTRODUCTION}

Linalool $\left(\mathrm{C}_{10} \mathrm{H}_{18} \mathrm{O}\right)$ is a hydrophobic and monoterpenoid compound which are extracted from plants for use as perfumes and flavouring food and beverages[1].

Revised Manuscript Received on December 30, 2019.

* Correspondence Author

Abhishruti Bhuyan*, Department of Electronics and Communication Engineering,Regional Institute of Science and Technology, Meghalaya793101 ,

Bipan Tudu, Department of Instrumentation and Electronics Engineering, Jadavpur University, Kolkata-700098, India

Rajib Bandopadhyay, Department of Instrumentation and Electronics Engineering, Jadavpur University, Kolkata-700098, India

Sudarshan Gogoi,Department of Chemical Science,

Amarprit Singh, Department of Electronics and Communication Engineering, Tezpur University,Tezpur-784028, India

(C) The Authors. Published by Blue Eyes Intelligence Engineering and Sciences Publication (BEIESP). This is an open access article under the CC BY-NC-ND license (http://creativecommons.org/licenses/by-nc$\underline{\mathrm{nd} / 4.0 /)}$
Detection and characterization of linallol are done by high performance liquid chromatography(HPLC),gas chromatography(GC) coupled to mass spectrometry or flame ionization detectors(FID) with different sample pretreatments[2-8]. These methods involve high cost method[6];instrumentation and they are not easily applicable in routine analysis. Literature available for detection of linalool are- GC-FID, GC-MS, and GC-olfactometry for linalool in the plant Michelia alba[2]; investigation of linalool using GC-FI, GC-MS, and GC-Olfactometry in white Austrian clover flowers[3]; quantitative analysis of linalool in wine[4]; determination of myristicin and linalool in plants exposed to microwave radiation byHPLC[5]; assay of linalool in coriander oil by proton NMR characterization and adulteration monitoring of linalool by Electrospray Ionization Mass Spectrometry[7].A new method based on fluidized-bed extraction combined with detection by GC-MS was developed to detect linalool [8]. Since the analytical methods as discussed above are not suitable for fast routine tests, electrochemical sensing has been established as a low cost and fast detection technique of odorant molecules such as a supported lipid bilayer membrane (s-BLMs) formed on a freshly cleaved metallic surface [9]. Although a variety of electrodes have been developed for electrochemical sensors using noble metals, carbon materials in Screen printed carbon electrodes (SPCE) deposited on rigid substrates to flexible materials like paper, fabric, wearable cloth etc have drawn attention. The SPCEs are becoming popular due to low manufacturing cost and their diverse design options using thick film technology. The SPCE features mixtures of two main components - carbon powder (graphite) acting as electroactive sensor and a liquid binder (lipophilic organic liquid). The organic liquid acts as a binder as well as influences the overall electrochemical behaviour of the paste [10]. It offers hydrophobic character of the carbon paste surface, which is the main reason for different behaviour of CPEs compared to carbon solid electrodes. In the last decade SPCEs has become popular for detection of various compounds[11-16] such as - a preanodized SPCE for propofol at $\mathrm{pH} 7.0$ of phosphate buffer solutions [11] ; a SPCE modified with the nanocomposite for nilutamide[12] ;graphite nanosheets and $\mathrm{ZnO}$ nanoparticles deposited SPCE for glucose oxidase[13]; Ag@GNRs film modified SPCE for pesticide methyl parathion [14]; a chitosan (CHT) crafted zinc $\mathrm{ZnO}$ nanoneedles modified SPCE for 4-nitrophenol in water[15]. Researchers have conducted experiment for comparing commercial SPCEs such as in a SPCE (C-110, Dropsens, Ltd. ,Spain) along with four other SPCE are tested to compare their performance[16]. 


\section{Preanodized Screen Printed Carbon Electrode for Detection of Linalool using Three Terminal Network}

In their study the authors estimated the best performance in C-110 as-anoidic and cathoidic peak current ratio: 1.18, $E_{p c}=0.112 \mathrm{~V}$, Sensitivity $=97 \mathrm{mV}, 20 \mu \mathrm{A} \mathrm{mM}{ }^{-1}$.

Motivated by the advantages of SPCEs, in this paper we present a preannodized commercial SPCE for detection of linalool and analysis of its model electrical parameters using three terminal analysis similar to C-110[17].

\section{Three terminal circuit analysis of SPCE}

Fletcher [18] suggested that although conventionally the three terminal sensors are represented by a two terminal circuit is better represented by a three terminal circuit as shown in Fig.1(a) where $R_{1}, R_{2}$ and $R_{3}$ are node to node resistances while $C_{4}, C_{5}$ and $C_{6}$ are three inter-electrode stray capacitances. In our earlier work we have proposed an alternative delta equivalent three terminal circuit which fits best to a higher order ( $2^{\text {nd }}$ and $3^{\text {rd }}$ ) model (Fig.1(b)) [17]. Further we consider that the basic Randle's circuit appears across WE and RE node with $\mathrm{R}_{\mathrm{d}}$ representing the analyte resistance, $R_{c}$ as the charge transfer resistance and $C_{6}$, the double layer capacitance. In this circuit we considered that the solution resistance between RE and CE is compensated by the potentiostat [19]. (a)

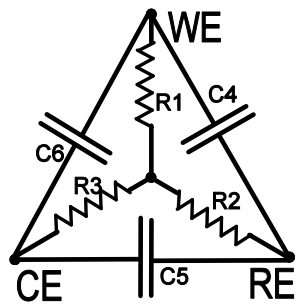

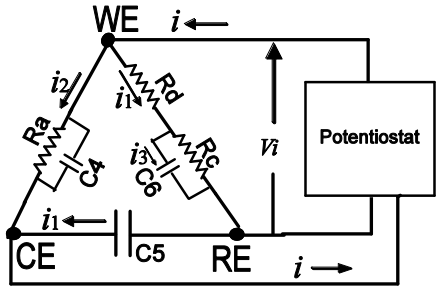

(b)
Fig.1 Three terminal electrode networks (a) Original

(b) proposed[14]

In the circuit of Fig.1(b) the network $R_{d}+\left(R_{c} . C_{6}\right)$ represents the WE impedance, while $R_{a}, C_{4}$ and $C_{5}$ represents interelectrode stray parasites.

In this paper we propose ARMAX model transfer functions $\mathrm{G}(\mathrm{s})=I(\mathrm{~s}) / V(\mathrm{~s})$ of the three terminal network of the SPCE in linalool as the analyte, where $I(\mathrm{~s})$ is the response current and $V(\mathrm{~s})$ is the applied voltage. From the transfer function model we derive the impedances $\mathrm{Z}(\mathrm{s})=$ $\mathrm{V}(\mathrm{s}) / \mathrm{I}(\mathrm{s})$ which are fitted to our three terminal impedance of $\operatorname{Eq}(1)$.

\section{A. ARMAX Modeling}

In our earlier work we have performed ARMAX modeling of five different metal electrodes of a custom made voltammetric e-tongue [20]. Motivated by the promising results on analysis of the performance of the five electrodes, in this paper we propose that ARMAX model can be estimated from the input and output cyclic voltammetry(CV) data of the SPCE.

We define a linear time-invariant (LTI) system with an input signal $u(n)$ and output $y(n)$ by eqn (1) as:

$$
\sum_{k=1}^{n a} a_{k} y(n-k)=\sum_{k=1}^{n b} b_{k} u(n-k)+e(n-k)
$$

where $e(n-k)$ is the moving average error, $n_{a}$ is the number of poles; $n_{b}$ is the number of zeroes and $k$ is the moving average time delay. We generate the data of length $n$ from measured input voltage $(u)$ and output response current $\left(i_{0}\right)$ of the SPCE from CV measurement given by :

$$
\begin{aligned}
& u(k)=[u(k-1) u(k-2) \ldots . . u(k-n)] \\
& i_{0}(k)=\left[i_{0}(k-1) i_{0}(k-2) \ldots . i_{0}(k-n)\right]
\end{aligned}
$$

The prediction error minimization (PEM) system identification algorithm minimizes a cost function given by Eqn(3).

where $\in(\mathrm{t})$ is given by:

$$
V_{N}(G, H)=\sum_{\mathrm{t}=1}^{n} \epsilon^{2}(t)
$$

$$
\in(t)=y(t)-\hat{y}(t)
$$

where $y(t)$ is the measured response current $i_{0}(\mathrm{t})$ and $\hat{y}(\mathrm{t})$ is the estimated response current $\widehat{\imath_{0}}(\mathrm{t})$ of the electrodes.

\section{II.MATERIALS AND METHODS}

The experiments were conducted in steps as stated below-

i) Preparation of solutions

ii) Cyclic voltammetry of the SPCE at different linalool concentrations and storing of input voltage and output current

iii) ARMAX modeling using CV data for different concentrations of linalool

iv) Fitting of ARMAX model to three terminal network

v) Impedance analysis

vi) Amperometric measurement

vii) Repeatability test

\section{B. Chemicals}

All of the chemicals used in this work were of analytical grade and were used without further purification. Standard solutions of linalool $\left(\mathrm{C}_{10} \mathrm{H}_{18} \mathrm{O}, 98 \%\right)$, sulfuric acid $\left(\mathrm{H}_{2} \mathrm{SO}_{4}-\right.$ 95-97\%) and potassium chloride $(\mathrm{KCl})$ were purchased from Sigma Aldrich (USA).

\section{Sensor and instrument}

The screen-printed electrodes(SE-100) were purchased from Zensor(USA). The Zensor screen printed SPE has a planar carbon working electrode (central circle) printed on a PVC substrate, a carbon auxiliary electrode (outer annular crescent) and $\mathrm{Ag} / \mathrm{AgCl}$ pellet reference electrode. In the SPCE relatively large tabs at the top of the SPE enable electrical contacts. Fig.2(a) shows the SPEC while Fig.2(b) shows the electrode dimensions. The surface of the SPCE was rinsed with ultrapure water between successive measurements. The SPCE was preannodized in a $0.05 \mathrm{~mol}$ $\mathrm{H}_{2} \mathrm{SO}_{4}$ solution by applying anodic potentials (ranging from -0.5 to $+0.5 \mathrm{~V}$ ) for five cycles with a scan rate of $10 \mathrm{mV} / \mathrm{s}$. A potentiostat (SP-300,BioLogic,France) was used for CV measurements of the SPCE. 


\section{Preparation of solution}

Potassium chloride solution ( $\mathrm{pH}$ 7.0) was prepared from crystalline $\mathrm{KCl}$ as the supporting electrolyte with a concentration of $0.1 \mathrm{M}$. Stock solution of $0.65 \mathrm{mM}$ linalool was prepared by dissolving $14.35 \mu \mathrm{l}$ linalool in $100 \mathrm{ml}$ water containing $0.1 \mathrm{M} \mathrm{KCl}$ by constant stirring. Similarly $0.325 \mathrm{mM}, 0.1625 \mathrm{mM}, 0.0812 \mathrm{mM}$ solutions were prepared by half diluting the stock solution successively while keeping the concentration of $\mathrm{KCl}$ constant. The cell and the SPCE were cleaned and washed thoroughly by water. We have used deionized water purified from a MilliQ purification system.

For ARMAX modeling of the SPCE we have used the time series input-output data of the CV for a total 2000 data points during one cycle(0-1-0 Volt) with a scan rate of $10 \mathrm{mV} / \mathrm{s}$. Therefore the input frequency of the CV is $20 \mathrm{~Hz}$.

\section{III.RESULTS AND DISCUSSIONS}

\section{A. CV peak currents with linalool concentration}

The sensitivity of the SPCE was evaluated from the CV current response with varying concentration of linalool. We have conducted CV with concentrations of $0.65 \mathrm{mM}$, $0.325 \mathrm{mM}, \quad 0.1625 \mathrm{mM}$ and $0.0812 \mathrm{mM}$ as shown in Fig.3(a),however below $0.0812 \mathrm{mM}$ the peaks are found to be noisy which are omitted. It is found that the peak oxidation current increases with concentration of linalool while the redox peak is almost constant. The oxidation peak currents for varying concentration at a voltage of $0.86 \mathrm{~V}_{\text {rms }}$ is shown in Fig.3(b). The quadratic regression equation for the concentration vs current relation is found as $i=12.9 c^{2}-$ $1.2 c+0.02$ with $r^{2}=0.9994\left(\mathrm{RMSE}=9.19 \times 10^{-8}\right)$, where $i$ is

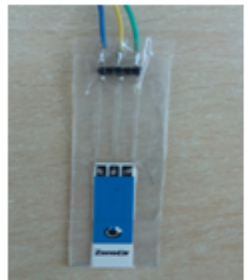

(a)

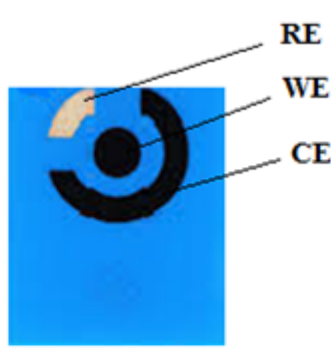

(b)
Fig.2 Zensor SPCE(SE-100) (a) after connecting with lead wires; package size: $25.4 \mathrm{~mm} \times 7 \mathrm{~mm} \times 0.625 \mathrm{~mm}$ (b) three electrodes of the SPCE ; central-WE, right-RE and circular-CE; exposed surface area of the WE- 0.0314 $\mathrm{mm}^{2}$ and CE- $0.1256 \mathrm{~mm}^{2}$

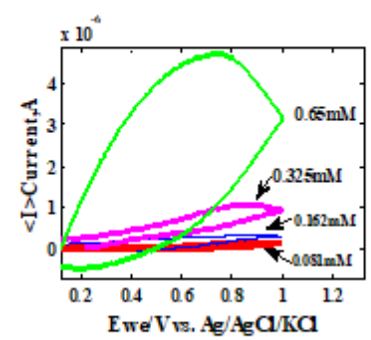

(a)

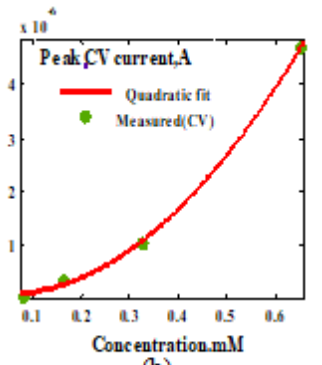

(b)
Fig. 3 Voltammograms of the SPCE $\left(10 \mathrm{mVs}^{-1}\right.$ scan rate) solutions of linalool (a) CV for one cycle each (b) variatio: peak current with concentration the peak current $\left(\times 10^{-6} \mathrm{~A}\right)$ and $c$ is the concentration of linalool in $\mathrm{mM}$. The highest ratio of peak anoidic and cathoidic current is found as 0.58 while the sensitivity is found as $0.25 \mu \mathrm{AmM}^{-1}$.

\section{B. Estimation of impedance}

The estimated transfer function $G(s)$ of the SPCE (Table-I) that fits with the circuit of Fig.1 (c) is of the form:

$$
G(s)=\frac{1+a_{1} s+a_{2} s^{2}+a_{3} s^{3}}{1+b_{1} s+b_{2} s^{2}}
$$

from which we get the impedance equation as:

$$
Z(s)=\frac{1+b_{1} s+b_{2} s^{2}}{1+a_{1} s+a_{2} s^{2}+a_{3} s^{3}}
$$

The transfer functions estimated for the SPCE shown in Table I indicate that for different concentrations of linalool the model parameters are different. The impedance of the SPCE at four different concentrations as shown in Table-1 were fitted to the three terminal impedance Eqn(1) to estimate the electrical parameters. The three electrode parameters $\left(R_{s o l}, R_{c t}\right.$ and $\left.C_{d l}\right)$ and the two parasitic parameters $\left(R_{p}\right.$ and $\left.C_{p}\right)$ estimated are shown in Fig.5 at various concentrations.

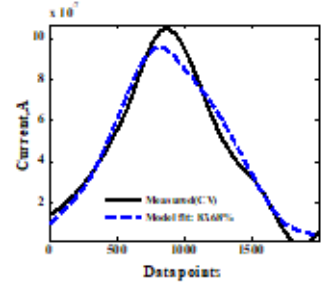

(a)

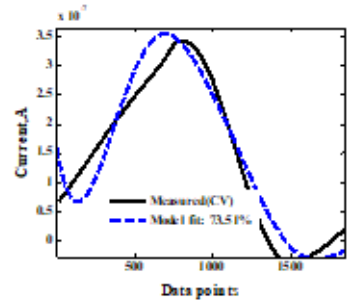

(c)

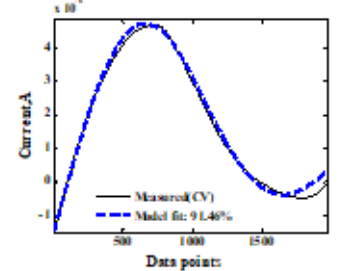

(b)

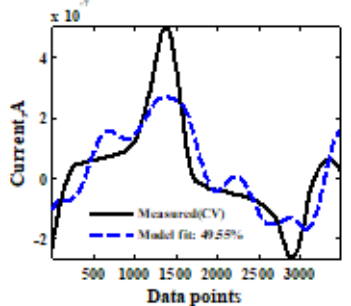

(d)
Fig.4 Model fits for the network (a) $0.0812 \mathrm{mM}$ (b) $0.1625 \mathrm{mM}$ (c) $0.325 \mathrm{mM}$ and (d) $0.65 \mathrm{mM}$

TABLE I

IMPEDANCE FUNCTIONS $Z(\mathrm{~s})$

\begin{tabular}{c|c|c|c|}
\hline $\begin{array}{c}C \\
(\mathrm{mM})\end{array}$ & $\mathbf{Z}(\mathrm{s})$ & Pole & Zero \\
\hline \multirow{2}{*}{0.0812} & $\frac{134.2(s+1.16)\left(s^{2}+0.21 s+22.74\right)}{(s-84.09)(s+0.22)}$ & $-0.0006+j 0.0094$ & -0.1274 \\
& $\frac{-0.0040}{(s+9.8)(s-0.14)}$ & -0.3650 & -9.8911 \\
\hline \multirow{2}{*}{0.1625} & $\frac{276.2(s+0.36)\left(s^{2}+0.013 s+0.019\right)}{(s+3)}$ & $-0.0068 \pm j 0.1387$ & 0.1482 \\
\hline \multirow{2}{*}{0.325} & $\frac{4167(s+0.37)\left(s^{2}-0.001 s+0.057\right)}{(s+10.59)(s+0.18)}$ & -0.0075 & -0.2117 \\
\hline \multirow{2}{*}{0.65} & $\frac{-4492.3\left(s^{2}-0.19 s+0.12\right)}{(s+1.5)}$ & $0.0000 \pm j 0.0048$ & -0.0036 \\
\hline
\end{tabular}



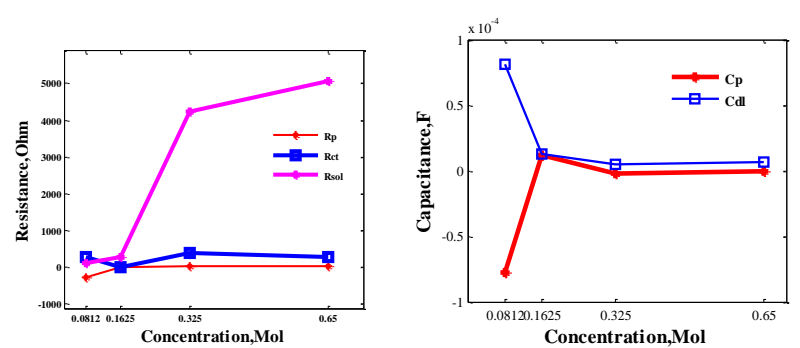

Fig. 5 Variations of electrical parameters with concentration of linalool at $0.0812 \mathrm{mM}$ to $0.65 \mathrm{mM}$ (a)

It is observed that the analyte resistance $R_{\text {sol }}$ increase to about $5 \mathrm{~K} \Omega$ when the concentration of linalool is increased from $0.081 \mathrm{mM}$ to $0.65 \mathrm{mM}$. Similarly the parasitic resistance $R_{p}$ also increases from $-266.62 \Omega$ to $19.82 \Omega$, while $R_{c t}$ has its own charge transfer resistance values of
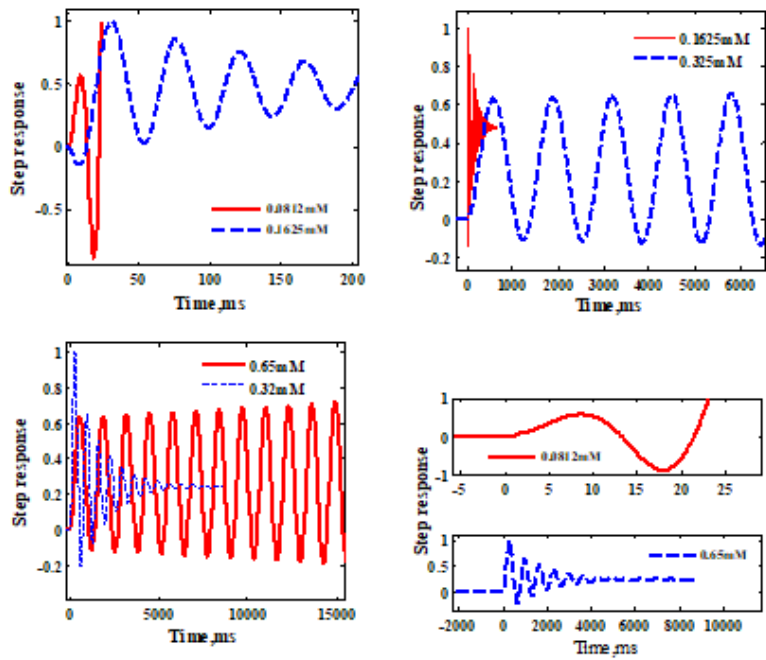

Fig.6 Comparison of step responses of the SPCE with higher concentration (a) $0.08125 \mathrm{mM}$ with $0.162 \mathrm{mM}$ (b) $0.1625 \mathrm{mM}$ with $0.32 \mathrm{mM}$ (c) $0.32 \mathrm{mM}$ with $0.65 \mathrm{mM}$ (d) ).0812 $\mathrm{mM}$ with $0.65 \mathrm{mM}$.

$266.79 \Omega, 15.66 \Omega, 401.48 \Omega$ and $276.78 \Omega$ respectively $\quad$.On the other hand the double layer capacitance $\left(C_{d l}\right)$ decreases from $81.1 \mu \mathrm{F}$ to $6.7 \mu \mathrm{F}$, while the parasitic capacitance $\left(C_{p}\right)$ monotonically decreases from $77.45 \mu \mathrm{F}$ to $0.37 \mu \mathrm{F}$. From this it is evident that the parasitic resistance increases while parasitic capacitance decreases as linalool concentration increases in the SPCE.

\section{Electrode stability}

Fig. 4 shows the fits of the models. It is observed that the fits for linalool concentrations of $0.0812 \mathrm{mM}, 0.1625 \mathrm{mM}$, $0.325 \mathrm{mM}$ and $0.65 \mathrm{mM}$ are- $83.68 \%, 91.46 \%, 73.5 \%$ and $49.55 \%$ respectively. The reason for low fit in highest concentration $(0.65 \mathrm{mM})$ is inconsistent $\mathrm{CV}$ current of the SPCE may be due to degradation of the reference electrode during reaction of the $\mathrm{Cl}^{-}$ion with linalool at higher concentration. The $\mathrm{Cl}^{-}$ions are formed due to $\mathrm{CV}$ at $1 \mathrm{~V}$ that reacts with linalool producing 10-chlorodecanoyl chloride:

$\mathrm{C}_{10} \mathrm{H}_{18} \mathrm{O}+\mathrm{Cl}_{2}=\mathrm{C} 10 \mathrm{H} 18 \mathrm{Cl}_{2} \mathrm{O}$. It may accumulate at the interface between electrode and electrolyte causing polarization and affects the Redox process. The unstability of the SPCE at higher concentration of linalool is also evident from the pole-zero positions (Table-1),the step response(Fig.6) and Nyquist plot(Fig.7). In Fig.6(a-c), the step responses are compared with the next higher concentration of linalool. It is observed from table 2 that as concentration increases the oscillatory response of the SPCE worsens. In Fig.6(b) the response at $0.162 \mathrm{mM}$ shows damped oscillations till $800 \mathrm{~ms}$ while at $0.325 \mathrm{mM}$ till $6 \mathrm{~s}$ and at $0.65 \mathrm{mM}$ till $1.5 \mathrm{~s}$ (Fig.6(c)). Fig.6(d) shows the comparison of step response of the SPCE at lowest $(0.0812 \mathrm{mM})$ and highest $(0.65 \mathrm{mM})$ concentrations. Although from the pole positions (Table-1) the SPCE apparently shows stable, however from the Nyquist plots (Fig.7) the stability criterion can be analyzed. If there is a pole in right half plane(RHP), for a system to be stable the following condition should be fulfilled:

$$
Z=N+P
$$

Where $\mathrm{Z}$ is the numbers of poles in RHP,P is the poles in the RHP and $\mathrm{N}$ is the numbers of clockwise encirclements of the $-1+\mathrm{j} 0$ point. From this condition we find that$0.08 \mathrm{mM}: Z=0, N=0$ and $P=0$ so the SPCE is stable $0.162 \mathrm{mM}: Z=1, N=2$ and $P=0$ so the SPCE is unstable

$0.32 \mathrm{mM}: Z=0, N=0$ and $P=1$ so the SPCE is unstable $0.65 \mathrm{mM}: Z=0, N=0$ and $P=1$ so the SPCE is unstable

The gain margins of the SPCE models are also found as- $128.56 \mathrm{~dB}, 91.13 \mathrm{~dB}, 94.00 \mathrm{~dB}$ and infinity at concentrations of $0.0812 \mathrm{mM}, 0.1625 \mathrm{mM}, 0.325 \mathrm{mM}$ and $0.65 \mathrm{mM}$. From this analysis it is evident that the SPCE shows an unstable model at higher concentration thereby producing inconsistent response.

\section{Impedance spectra}

The impedance behavior has been verified by impedance spectra as shown in Fig.8. Distinct impedance semicircles are observed at different quadrants $(Q)$ specified as:

0.081mM: $Q, I I I(-R-j X)$

TABLE II

STABILITY AND IMPEDANCE PARAMETERS

\begin{tabular}{ccccccc}
\hline $\begin{array}{c}\text { Conc } \\
(\mathrm{mM})\end{array}$ & $\begin{array}{c}\text { Oscillation } \\
\text { time(ms) }\end{array}$ & $\mathrm{P}$ & $\mathrm{Z}$ & $\mathrm{N}$ & $\begin{array}{c}\text { Gain } \\
\text { margin } \\
(\mathrm{dB})\end{array}$ & Impedance \\
\hline $\mathbf{0 . 0 8 1}$ & 17 & 0 & 0 & 0 & 128.56 & $-R+j X$ \\
\hline $\mathbf{0 . 1 6 2}$ & 800 & 0 & 1 & 2 & 91.13 & $+R+j X$ \\
\hline $\mathbf{0 . 3 2}$ & 6000 & 1 & 0 & 0 & 94.00 & $-R-j X$ \\
\hline $\mathbf{0 . 6 5}$ & 1500 & 1 & 0 & 0 & Inf & $-R-i X$.
\end{tabular}




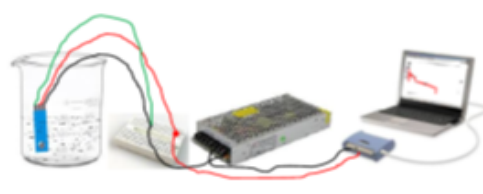

(a)

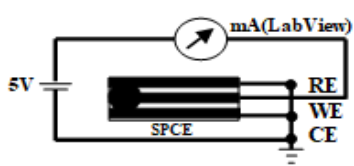

(C)

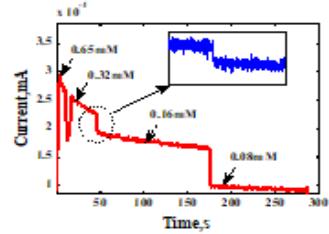

(b)
Fig.9 Amperometric measurement of the SPCE to linalool (a) schematic diagram of the setup. The WE is supplied with $+5 \mathrm{~V}$ and the RE-CE are grounded.The current through the WE is measured using a current channel of the DAQ card (b) measuring circuit (c) acquired current signal at successive dilution of the concentration. The inset figure shows the noise

$0.162 \mathrm{mM}: Q, I(R+j X)$

$0.32 \mathrm{mM}: Q, I I I(-R-j X)$,

$0.65 \mathrm{mM}: Q, I I I(-R-j X)$ and $Q, I V(-R-j X)$

where $R$ and $X$ are the resistance and reactance of the SPCE respectively.

Prominent negative semicircles are seen in- third and fourth quadrants at $0.65 \mathrm{mM}, 0.325 \mathrm{mM}, 0.0812 \mathrm{mM}$ due to

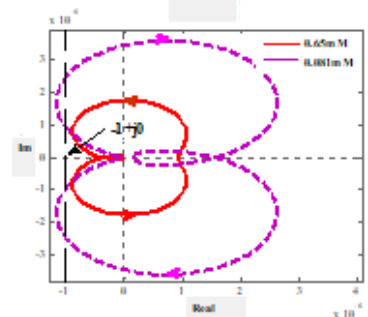

(a)

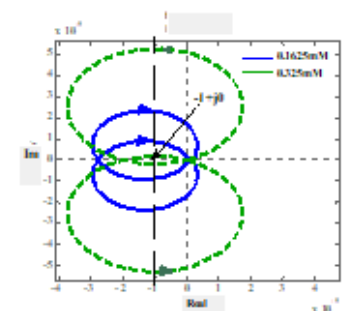

(b)
Fig.7 Nyquist plot of models of the SPCE. Due to better visibility the plots are shown for two pairs having equal scale values (a) at $0.065 \mathrm{mM}$ and $0.0812 \mathrm{mM}$ and (b) at $0.1625 \mathrm{mM}$ and $0.325 \mathrm{mM}$

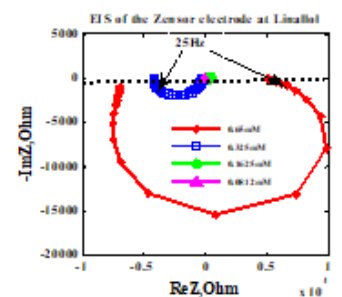

(a)

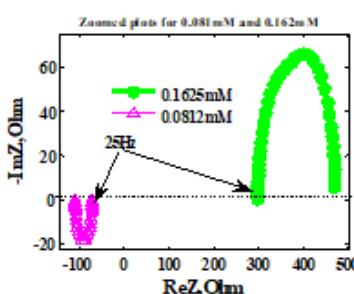

(b)
Fig.8 Impedance spectra of SPCE (a) at $0.081-0.65 \mathrm{mM}$ (b) Zoomed for 0.0812 and $0.1625 \mathrm{mM}$; The impedance intercepts at $25 \mathrm{~Hz}$ is shown to relate to $\mathrm{CV}$ scan rate of $\mathbf{2 0 H z}$

negative $C_{p}$ and $R_{p}$ while at $0.1625 \mathrm{mM}$ the semicircle is positive at first quadrant since all electrical parameters are positive. From the impedance spectra the real part of the impedances of the SPCE are found -109 $\Omega, 300 \Omega,-4113 \Omega$ and $5122 \Omega$ at $0.0812 \mathrm{mM}, 0.1625 \mathrm{mM}$,
$0.325 \mathrm{mM}$ and $0.65 \mathrm{mM}$ respectively. The CV scan rate used in our experiment is equivalent to $20 \mathrm{~Hz}$ as discussed in section 2. However at nearest discrete frequency of $25 \mathrm{~Hz}$ (shown by arrow) in impedance spectra indicates that negative electrical parameters cannot be avoided by changing the scan rate since neither of the spectra crosses zero-axis in both real and imaginary impedance.

\section{E. Amperometric analysis}

From the $\mathrm{CV}$ and impedance spectra it is evident that the SPCE is able to detect linalool to a lowest level of 0.0812mM.We have used an amperometric circuit(Fig.9(a) and (c)) for sensing linalool using the three electrode SPCE based on a data acquisition(DAQ) card connected to a PC with LabView. The SPCE was used in two terminal mode by connecting the RE to the CE. The current flowing through the circuit on application of a voltage of $1 \mathrm{~V}$ was measured by the DAQ card using LabView. Stock solution of linalool was prepared in a borosil beaker of $500 \mathrm{ml}$ containing $0.1 \mathrm{M} \mathrm{KCl}$ by diluting the stock solution successively as discussed in section II. The noise in the current signal was filtered by configuring a Butterworth filter in Labview. The acquired signal is shown in Fig.9(b). It is observed that the current monotonically decreases as $0.29 \mathrm{~mA}, \quad 0.26 \mathrm{~mA}, 0.16 \mathrm{~mA}$ and $0.09 \mathrm{~mA}$ when the concentration is decreased successively as- $0.65 \mathrm{mM}$, $0.325 \mathrm{mM}, 0.16 \mathrm{mM}$ and $0.0812 \mathrm{mM}$.From this measurement the sensitivity of the SPCE to linalool is found as $0.3289 \mathrm{~mA} / \mathrm{mM}$. This current can be converted to get a sensitivity of $328.9 \mathrm{mV} / \mathrm{mM}$ across a load resistor of $1 \mathrm{~K} \Omega$.

To test the repeatability of the sensor the sensor responses over ten repeated trials on $0.325 \mathrm{mM}$ linalool were observed as shown in Fig.10(a). The sensor was dipped and removed ten times over a period of $5 \mathrm{~min} 30$ s period and the responses were recorded. The data during each response period was processed to determine the mean and the standard deviation. The errorbar plot shown in Fig.10(b) indicated a maximum deviation of $0.04 \mu \mathrm{A}$ in fifth trial which is almost $1.2 \%$ of the mean value. Therefore the SPCE is able to show a good repeatability in sensing linalool.

\section{IV.CONCLUSION}

In this work, a preannodized commercial SPCE in three terminal network analysis has been used for the first time to
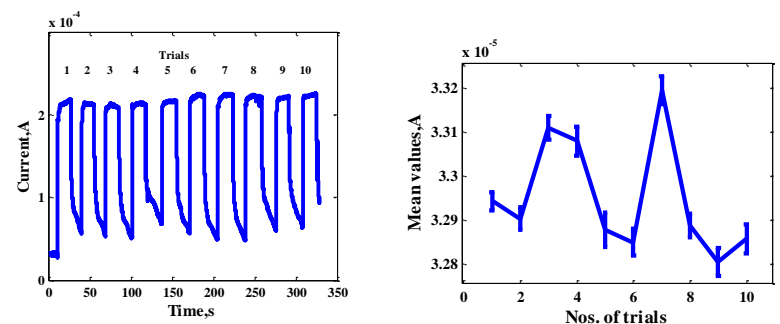

Fig.10 Repetition measurement (a) the SPCE response to ten trials with a concentration of $0.325 \mathrm{mM}$ of linalool (b) error bar plot over the mean values of the responses

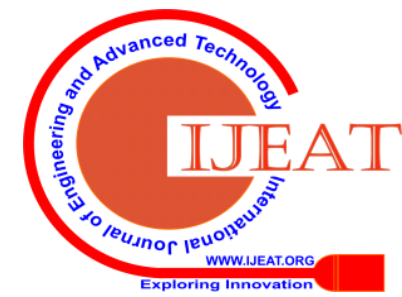




\section{Preanodized Screen Printed Carbon Electrode for Detection of Linalool using Three Terminal Network}

detect linalool concentration quantitatively. The study shows that the SPCE can detect linalool in two terminal mode also with a linear current sensitivity. The three terminal analysis shows that the parasitic resistance increases while the parasitic capacitance decreases with linalool concentration. The amperometric measurement method shows a good sensitivity and repeatability of the SPCE. The proposed method is useful for detection of linalool having great importance in food and chemical industries.

\section{REFERENCES}

1. Alberto Cantalapiedra, Ma Jesffls Gismera, Ma Pilar da Silva, Jesffls R. Procopio, Teresa Sevilla, Evaluation of Different Electrochemical Transducers Based on Polystyrene Sulfonate - Carbon Composites with Nickel Catalyst for Rapid Detection of Fragrance Allergens, Electroanalysis, 2013, 25, No. 1, $243-251$

2. En-Qin Xia, Yang Song, Xu-Xia Ai,Ya-Jun Guo, Xiang-Rong $\mathrm{Xu}$ and Hua-Bin Li, A New High-Performance Liquid Chromatographic Method for the Determination and Distribution of Linalool in Michelia alba, Molecules. $2010 \quad$ Jul; $15(7): \quad 4890$ 4897,doi: 10.3390/molecules15074890

3. Gerhard Buchbauer, Leopold Jirovetz, Alexej Nikiforov, Comparative Investigation of Essential Clover Flower Oils from Austria Using Gas Chromatography-Flame Ionization Detection, Gas Chromatography-Mass Spectrometry, and Gas Chromatography-Olfactometry, J. Agric. Food Chem.1996,447,18271828

4. D. Sejer Pedersen,Dimitra L. Capone,George K. Skouroumounis,Alan P. Pollnitz,Mark A. Sefton, Quantitative analysis of geraniol, nerol, linalool, and $\alpha$-terpineol in wine, Analytical and Bioanalytical Chemistry, February 2003, Volume 375, Issue 4, pp 517-522

5. Ildikó Lung, Manuela Stan, Ocsana Opriș \& Maria-Loredana Soran, Determination of Myristicin and Linalool in Plants Exposed to Microwave Radiation by High-Performance Liquid Chromatography, Analytical Letters, Volume 48, 2015 - Issue 4

6. Humeida A. El-obeid, Jabir S. Mossa \& Mahmoud M. A. Hassan, Proton NMR Assay of Essential Oils VIII. Assay of Linalool in Coriander Oil, Analytical Letters, Volume 15, 1982 - Issue 9

7. Rita C. Z. Souza, Marina Marques Eiras, Elaine C. Cabral,Lauro E. S. Barata, Marcos N. Eberlin \&Rodrigo R. Catharino, The Famous Amazonian Rosewood Essential Oil: Characterization and Adulteration Monitoring by Electrospray Ionization Mass Spectrometry Fingerprinting, Analytical Letters, Vol-44,Issue 15,2011

8. Karel Štěrba, Pavel Čejka, Jiří Čulík, and Marie Jurková, Karel Krofta, , Saaz, Kadanska , Alexander Mikyška and Jana Olšovská, Determination of Linalool in Different Hop Varieties Using a New Method Based on Fluidized-Bed Extraction with Gas Chromatographic-Mass Spectrometric Detection, J. Am. Soc. Brew. Chem. 73(2):151-158, 2015

9. Szyman'ska , H. Radecka , J. Radecki D. Kikut-Ligaj, Fullerene modified supported lipid membrane as sensitive element of sensor for odorants , Biosensors \& Bioelectronics 16 (2001) 911-915

10. Ivan Svancara and Klemens Schachl, Testing of unmodified carbon paste electrodes, Chem. Listy 93, 490 - 499,1999

11. Soundappan Thiagarajan \& Ching-Yi Cheng \& Shen-Ming Chen \& Tsung-Hsuan Tsai, Electrochemical detection of propofol at the preanodized carbon electrode, Journal of Solid State Electrochemistry, Volume 15, Issue 4, pp 781-786

12. Raj Karthik1 \& Natarajan Karikalan1 \& Shen-Ming Chen1 \& Periyasami Gnanaprakasam \& Chelladurai Karuppiah ,Voltammetric determination of the anti-cancer drug nilutamide using a screen-printed carbon electrode modified with a composite prepared from $\beta$ cyclodextrin, gold nanoparticles and graphene oxide, Microchimica Acta, Volume 184, Issue 2, pp 507-514,February 2017

13. Chelladurai Karuppiah \& Selvakumar Palanisamy ,Shen-Ming Chen \& Vediyappan Veeramani \& Prakash Periakaruppan , Direct electrochemistry of glucose oxidase and sensing glucose using a screen-printed carbon electrode modified with graphite nanosheets and zinc oxide nanoparticles, Microchimica Acta, , Volume 181, Issue 1516, pp 1843-1850 , November 2014

14. Mani Govindasamy, Veerappan Mani1, Shen-Ming Chen, Tse-Wei Chen \& Ashok Kumar Sundramoorthy,Methyl parathion detection in vegetables and fruits using silver@graphene nanoribbons nanocomposite modified screen printed electrode, Scientific Reports, volume 7, Article number: 46471,2017
15. Balamurugan Thirumalraj, Chellakannu Rajkumar, Shen-Ming Chen, Kuan-Yu Lin ,Determination of 4-nitrophenol in water by use of a screen-printed carbon electrode modified with chitosan-crafted $\mathrm{ZnO}$ nanoneedles, Journal of Colloid and Interface Science, Volume 499, 1 August 2017, Pages 83-92

16. Mistry, Kalyan Kumar; Kashyup, Sneha; Roy Chaudhuri, Chirasree; Saha, Hiranmay, A Comparative Study in Electrochemical Response of Some Commercial Screen-Printed Electrodes (SPEs) IEEE,Sensor Letters, American Scientific Publishers, Volume 12, Number 12, December 2014, pp. 1750-1759(10)

17. Abhishruti Bhuyan, Bipan Tudu ,Rajib Bandopadhyay, Arunangshu Ghosh , Sanjeev Kumar, Extended Kalman Filtering for Estimation of Parasitic Artifacts in Three Electrode Electrochemical Sensors, IEEE Sensors Letters ,2019, DOI: 10.1109/LSENS.2019.2943503

18. Stephen Harvey Fletcher, The two-terminal equivalent network of a three-terminal electrochemical cell, Electrochemistry Communications, Volume 3, Issue 12 , Pages 692-696 ,December 2001

19. Dimitrios K. , Kampouris, Xiaobo Ji,, Edward P. Randviir and Craig E. Banks, "A new approach for the improved interpretation of capacitance measurements for materials utilised in energy storage" ,RCS Advances,2015,5,12782-12791

20. Abhishruti Bhuyan ; Bipan Tudu ; Rajib Bandyopadhyay ; Arunangshu Ghosh ; Sanjeev Kumar, ARMAX Modeling and Impedance Analysis of Voltammetric E-Tongue for Evaluation of Infused Tea,IEEE Sensors Journal, Vol-19,Issue-11, 1558-1748,June,2019.

\section{AUTHORS PROFILE}

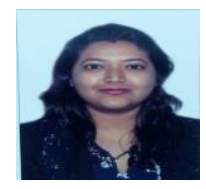

Abhishruti Bhuyan received the M.Tech degree from SRM University, Chennai, India in 2013. She is currently pursuing the Ph.D degree with the Department of Instrumentation and Electronics Engineering, Jadavpur University, Kolkata, India. She is currently an Assistant Professor with the Department of Electronics and Communication Engineering, Regional Institute of Science and Technology, Meghalaya, India.

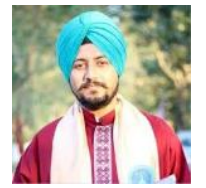

Bipan Tudu received the Ph.D. degree from Jadavpur University, Kolkata, India, in 2011. He is currently a Professor with the Department of Instrumentation and Electronics Engineering, Jadavpur University. His research interests include pattern recognition, artificial intelligence, machine olfaction, and the electronic tongue.

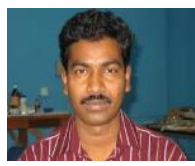

Rajib Bandyopadhyay received the Ph.D degree from Jadavpur University, Kolkata, India, in 2001.He is currently a Professor with the Department of Instrumentation and Electronics Engineering, Jadavpur University and a Research Professor with the Laboratory of Artificial Sensory Systems, ITMO University, Saint Petersburg, Russia. His research interests include the fields of machine olfaction, electronic tongue, and intelligent systems.

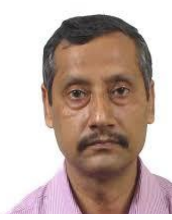

Sudarshan Gogoi received his M.Sc. degree from Tezpur University, India in 2014. He is currently pursuing the Ph.D degree with the Department of Chemical Science, Tezpur University, India. He received the Junior Research Fellowship(JRF) from the University Grants Commission (UGC),Govt. of India in2015.

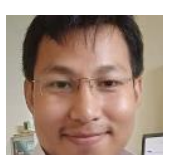

Amarprit Singh received the M.Tech degree from Tezpur University, India in 2018. He is currently pursuing the Ph.D degree with the Department of Electronics and Communication Engineering, Tezpur University, India. He has received the Inspire Fellowship from Department of Science and Technology (DST) Govt. of India in 2019. 